\title{
Single-Electron Population and Depopulation of an Isolated Quantum Dot Using a Surface-Acoustic-Wave Pulse
}

\author{
M. Kataoka, R. J. Schneble, A. L. Thorn, C. H. W. Barnes, C. J. B. Ford, D. Anderson, G. A. C. Jones, I. Farrer, \\ D. A. Ritchie, and M. Pepper \\ Cavendish Laboratory, J.J. Thompson Avenue, Cambridge CB3 OHE, United Kingdom
}

(Received 30 August 2006; published 22 January 2007)

\begin{abstract}
We use a pulse of surface acoustic waves (SAWs) to control the electron population and depopulation of a quantum dot. The barriers between the dot and reservoirs are set high to isolate the dot. Within a time scale of $\sim 100 \mathrm{~s}$ the dot can be set to a nonequilibrium charge state, where an empty (occupied) level stays below (above) the Fermi energy. A pulse containing a fixed number of SAW periods is sent through the dot, controllably changing the potential, and hence the tunneling probability, to add (remove) an electron to (from) the dot.
\end{abstract}

Coherent manipulation of confined quantum systems has significant importance for the development of quantum information technology [1]. Recent experiments on the charge or spin states of electrons in semiconductor quantum dots [2-6] have demonstrated quantum-logic-gate operations [7], and have given insight into the decoherence mechanisms. In these experiments, fast device operation at the nanosecond time scale is vital as manipulations need to be completed within the phase-coherence time. The electron population is normally controlled by pulsing a gate or source-drain voltage to move a dot level below or above the Fermi energy of the reservoirs, and waiting for an electron to tunnel through a barrier. This means that the tunnel barrier needs to be weak enough for electron tunneling to occur in a short time, although such a strong coupling between the dot and the reservoirs may not be ideal for decoupling the dot from the environment [8]. One method to avoid this is to pulse the tunnel-barrier gate to lower the barrier potential only when electron tunneling is required. However, cross coupling between the gates and the dot potential may make it necessary to pulse another gate simultaneously to keep the dot level constant.

As an alternative, we use a pulse of surface acoustic waves (SAWs) [9] to populate or depopulate a quantum dot that is well isolated from electron reservoirs. We prepare the dot in a nonequilibrium state where an empty (or occupied) dot level stays below (above) the Fermi energy of the reservoirs. In this arrangement, with a strong barrier potential the dot maintains its charge nonequilibrium state for more than $100 \mathrm{~s}$ [10]. A single pulse containing a few hundred thousand periods of a SAW is then sent through the dot. The piezoelectric potential associated with the SAW perturbs the dot potential, enhancing the tunneling probability [11]. The enhancement is still small, so that electrons fail to tunnel through the barrier in most of the cycles. Repeated attempts (for long enough pulse width) ensure one tunneling event during the pulse. Once one electron enters/escapes the dot, Coulomb blockade prevents tunneling of another electron. The change in the electron number is detected by the nearby quantum point contact [12].

The sample was fabricated from a GaAs/AlGaAs heterostructure with a two-dimensional electron gas (2DEG) $97 \mathrm{~nm}$ below the surface. The 2DEG density was $1.8 \times$ $10^{15} \mathrm{~m}^{-2}$ and the electron mobility was $160 \mathrm{~m}^{2} / \mathrm{V} \mathrm{s}$ at $1.5 \mathrm{~K}$. A quantum dot and a detector quantum point contact were defined by surface $\mathrm{NiCr} / \mathrm{Au}$ gates. A schematic sample diagram and a scanning electron microscopy (SEM) image of a device similar in design to the one used in the measurements are shown in Fig. 1(a). The lithographic size of the quantum dot is $350 \mathrm{~nm}$. A voltage of $-0.5 \mathrm{~V}$ was applied to the separation gate to isolate the

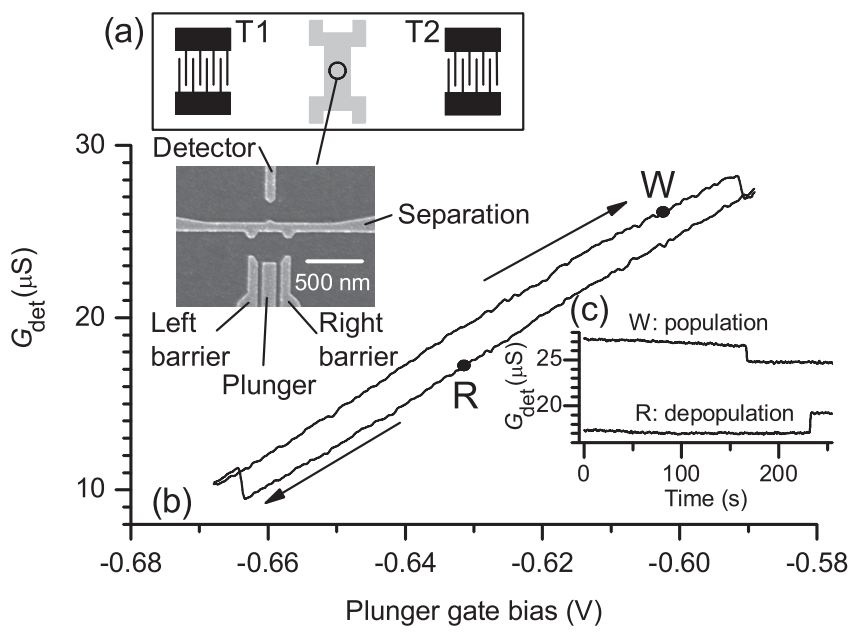

FIG. 1. (a) A schematic sample diagram and a SEM image of a device similar in design to the one used in the measurements. (b) The detector conductance $G_{\text {det }}$ as a function of plunger gate bias, showing a hysteresis loop. The sweep directions are marked by arrows. The points marked by $\mathrm{R}$ and $\mathrm{W}$ are where the plunger gate bias is set for depopulation and population measurements, respectively. (c) The lower and upper curves are the time dependence of $G_{\text {det }}$ after the plunger gate bias is set at the positions $\mathrm{R}$ and $\mathrm{W}$, respectively. 
detector circuit and the quantum-dot circuit. Two interdigital transducers (T1 and T2) each with 70 pairs of fingers were placed on the etched surface $2.5 \mathrm{~mm}$ away to the left (T1) and right (T2) of the quantum dot. The pattern is oriented so that SAWs travel parallel to the [011] direction. The period of the transducer fingers is $1 \mu \mathrm{m}$, and the resonant frequencies at $0.3 \mathrm{~K}$ were $2.7725 \mathrm{GHz}$ for $\mathrm{T} 1$ and $2.7714 \mathrm{GHz}$ for T2. An Agilent 8648D signal generator with pulse-modulation option was used to apply power to the transducer. A Tektronix PG5110 pulse generator was used for pulse modulation. The conductance through the detector circuit was measured using a standard lock-in technique. Measurements were performed at $0.3 \mathrm{~K}$. The electron temperature of the 2DEG, estimated from the width of the conductance resonance through the dot [13], was $0.5 \mathrm{~K}$ [14].

The quantum dot was set up with large potential barriers defined by the left and right barrier gates, so that the conductance through the dot was negligible. The charge state of the dot was monitored by measuring the conductance $G_{\text {det }}$ through the detector constriction. When the number of electrons in the dot changes by one as the plunger gate is swept, a step appears in the detector signal due to the capacitive coupling between the dot and the detector [12]. If electrons are able to tunnel through the barriers within the time constant of the measurement, charge equilibrium always appears to be maintained and electron population or depopulation occurs each time a dot level crosses the Fermi energy of the reservoirs. We raise the tunnel barrier even further, so that even when an occupied level is aligned with the Fermi energy, the electron stays in the dot for more than $100 \mathrm{~s}$ [10]. Dot depopulation can then occur in two ways. Either one waits for many seconds, or the occupied level needs to be set to a higher energy where the saddlelike barrier potential is weaker. Once the level is depopulated, in order to repopulate it the plunger bias needs to be made much more positive to weaken the tunnel barriers. This brings the level well below the Fermi energy. As a result, a hysteresis loop appears as the plunger is swept up and down at a moderate sweep rate $(\sim 5 \mathrm{~V} / \mathrm{h})$ [see Fig. 1(b)]. In this example, the plunger bias needs to be swept to below $-0.66 \mathrm{~V}$ for the electron to escape. In order to populate the same level, the plunger needs to be increased to $-0.59 \mathrm{~V}$ (the background change in $G_{\mathrm{det}}$ is due to a weak capacitive coupling between the plunger gate and the detector constriction).

We introduce the following initialization plunger sweeps to set the dot to a nonequilibrium charge state. To set a populated level above the Fermi energy, the plunger gate bias is first swept to near the negative end of the hysteresis loop $(-0.66 \mathrm{~V}$ in this case). After waiting for $\sim 10 \mathrm{~s}$ to ensure that the level is unoccupied, the plunger is swept to the positive end $(-0.59 \mathrm{~V})$, waiting for another $\sim 10 \mathrm{~s}$ to ensure that the level is populated. The plunger is then quickly swept to a final voltage [e.g., point " $R$ " in Fig. 1(b)]. We find that this routine reliably sets just one extra electron in the dot regardless of its initial status. The initialization procedure for setting an unoccupied level below the Fermi energy is to sweep the plunger to the positive end first, then to the negative end, and then to a final voltage [e.g., point "W" in Fig. 1(b)]. The lower and upper curves in Fig. 1(c) show $G_{\text {det }}$ as a function of time after the plunger gate bias is set to $-0.63 \mathrm{~V}$ and $-0.6 \mathrm{~V}$ respectively [marked by R and W in Fig. 1(b)] following an initialization sweep. These curves show that it takes $\sim 100 \mathrm{~s}$ for the electron to tunnel out from or into the dot under these conditions.

Population of the dot by a SAW pulse is performed by applying a pulse of microwave power $-2.1 \mathrm{dBm}$ with width $w_{p}=100 \mu \mathrm{s}$ at the resonant frequency to transducer T1 $1.5 \mathrm{~s}$ after an unoccupied level is initialized to a particular energy using the plunger bias (see Fig. 2). The time SAWs take to travel between the transducer and the dot is $\sim 900 \mathrm{~ns}$ and negligible on the time scale of our measurements. Also, because the pulse is much shorter than the measurement time constant, the direct effect of the SAW on the detector conductance [15] is negligible. In Figs. 3(a) and 3(b) the time derivative of the detector conductance $\left(d G_{\mathrm{det}} / d t\right)$ is plotted as a function of time and plunger bias. Figure 3(a) plots all the curves on the same scale, and clearly shows three heights of steps in $G_{\text {det }}$, labeled as " $-e$ ", " $+e$ ", and " $+2 e$ ". This notation refers to the charge state of the dot relative to its equilibrium population. The label $-e$ indicates that the dot is populated by one extra electron, and $+e$ or $+2 e$ denote that the dot is depopulated by one or two electrons, respectively. The majority of the peaks or dips in $d G_{\text {det }} / d t$ coincide with the SAW pulse, with a constant delay of $\sim 1 \mathrm{~s}$ due to the time constant $500 \mathrm{~ms}$ of the measurements. A small number of peaks or dips observed at different positions in time are due to a spontaneous change in the dot population.

Figure 3(b) shows that the majority of single-electron population signals $-e$ are clustered around $-0.6 \mathrm{~V}$ in plunger bias [marked by the dashed line, corresponding to W in Fig. 1(b)]. This is where the dot can be considered to be in "Write" mode (where we can add one electron to the dot with a SAW pulse). Between -0.605 and $-0.64 \mathrm{~V}$, a SAW pulse does not affect the dot population. Beyond $-0.64 \mathrm{~V}$, a SAW pulse removes typically one electron

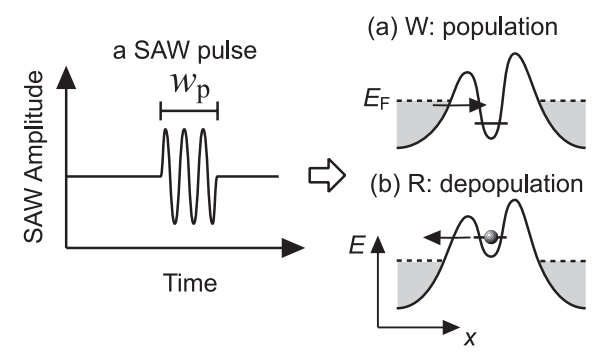

FIG. 2. (a) For population, a SAW pulse is sent to the dot initialized with an unoccupied level below the Fermi energy $E_{F}$ of the reservoirs. (b) For depopulation, an occupied level is initialized above $E_{F}$ before a SAW pulse is sent. 


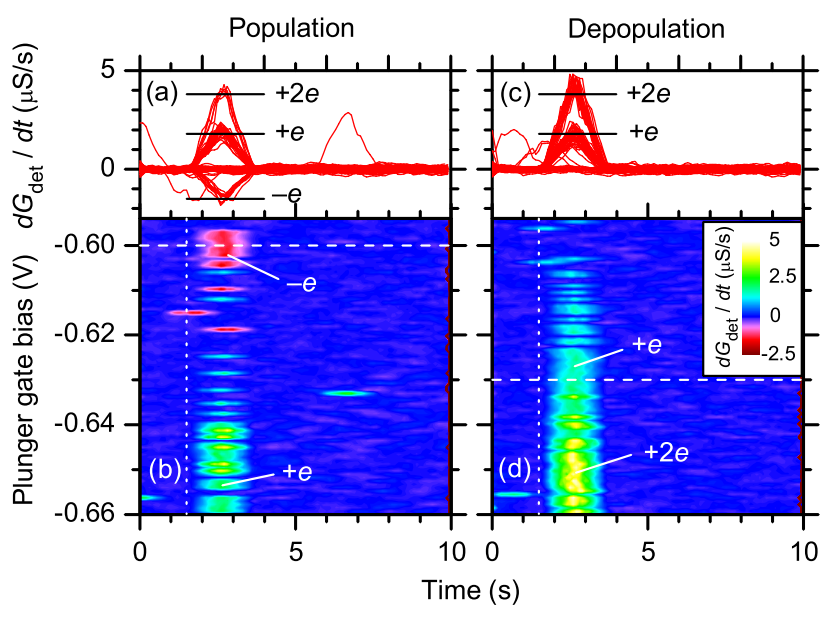

FIG. 3 (color online). The derivative of the detector conductance with respect to time, as a function of time after an initialization sweep. The final plunger bias is incremented between the time sweeps. Each trace in (a) and (b) is taken after an initialization sweep for population (see the main text for detail). In (a) all the curves are plotted on the same scale. The dips (denoted as $-e$ ) appear when one electron is added to the dot. The two heights of the peaks (denoted as $+e$ and $+2 e$ ) appear when one or two electrons are removed, respectively. Most of the signals coincide with the SAW pulse [its position is marked by the vertical dotted line in (b)] with a constant delay of $\sim 1 \mathrm{~s}$ due to the time constant of the measurements. The horizontal dashed line marks the plunger bias where the population by a SAW pulse is most reliable, corresponding to $\mathrm{W}$ in Fig. 1(b). Each trace in (c) and (d) is taken after an initialization sweep for depopulation. The horizontal dashed line marks the plunger bias where singleelectron depopulation is most reliable, corresponding to $\mathrm{R}$ in Fig. 1(b).

$(+e)$, and occasionally two electrons $(+2 e)$ from the dot. The $+e$ signal is due to the removal of an electron in the next occupied level (lower in energy) as this level is also set above the Fermi energy in this plunger bias range. In addition, when the initialization sweep occasionally fails to remove the electron in the highest occupied state, two electrons may be removed from the dot by a SAW pulse giving rise to a $+2 e$ signal.

Depopulation of the dot with an excess electron by a SAW pulse can be performed in a similar manner, as shown in Figs. 3(c) and 3(d). Here, the dot is initialized with one excess electron. No population signal $(-e)$ appears throughout the plunger bias range as the level is already occupied, and a large charging energy must be overcome if an incoming electron is to be added [16]. Single-electron depopulation signals $+e$ appear below $-0.61 \mathrm{~V}$, with a good success rate around $-0.63 \mathrm{~V}$ [marked by the dashed line, corresponding to $\mathrm{R}$ in Fig. 1(b)]. This can be considered to be the "Read" position, where one electron can be removed by a SAW pulse. Beyond $-0.64 \mathrm{~V},+2 e$ signals appear due to the removal of two electrons.

In order to investigate the population and depopulation mechanisms, we study their success rates. In experiments such as those shown in Fig. 3, the reliability of the initialization procedure affects the outcome of the switching. In order to remove this effect, after the dot is initialized, SAW pulses are repeated every $5 \mathrm{~s}$ while the plunger is switched between the Read and Write positions [" $R$ " and "W" in Fig. 1(b)] in between the pulses. Figs. 4(a) and 4(b) show the detector conductance and its derivative with respect to time, respectively, for such measurements. For example, if the previous pulse for population fails, the next pulse for depopulation is expected to fail as the dot does not have the extra electron needed for depopulation. Also, occasionally the dot occupation switches before the SAW pulse arrives [as shown by the arrow in Fig. 4(b)]. We exclude such cases from the statistics.

The pulse-width dependences of the success rates of population and depopulation out of $\sim 60$ possible events are plotted in Figs. 4(c) and 4(d), respectively. The fall of the success rates for $w_{p}$ below $20 \mu$ s for population and below $50 \mu \mathrm{s}$ for depopulation indicates low probabilities of population $P_{\text {pop }}$ and depopulation $P_{\text {depop }}$ per SAW cycle
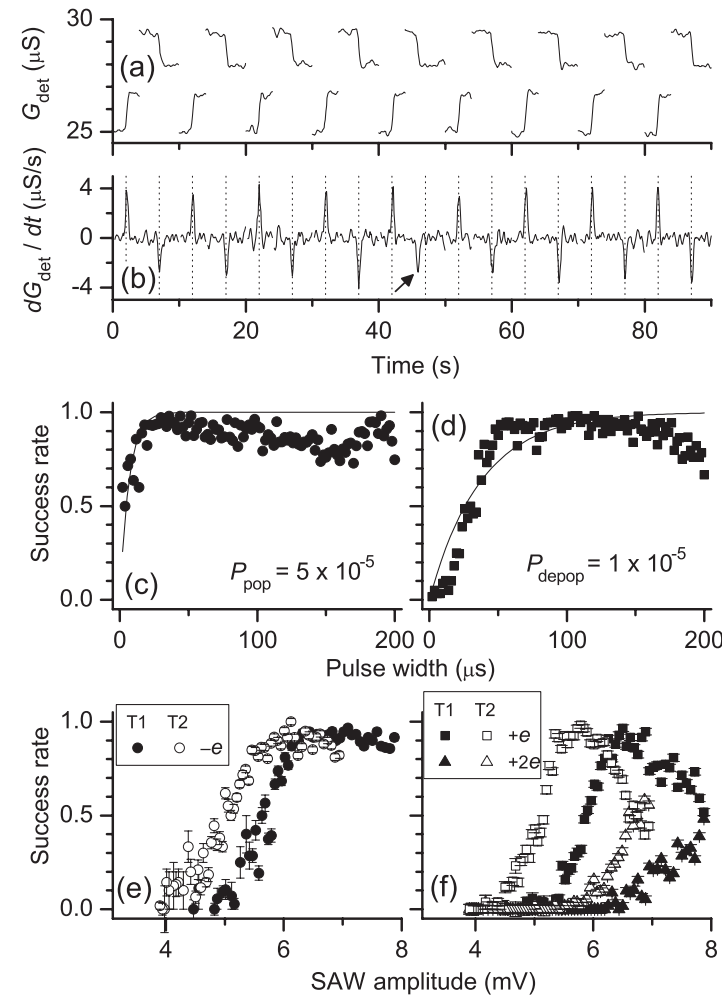

FIG. 4. (a) Switching measurements used for taking statistics of the success rates for population and depopulation. SAW pulses are repeated every $5 \mathrm{~s}$, while the plunger is switched between the R and W positions in Fig. 1(b) in between pulses. (b) The derivative of the detector conductance in (a). The vertical dotted lines are plotted every $5 \mathrm{~s}$. The arrow marks a switching event that occurred prior to the SAW pulse. (c) and (d) The success rates of population and depopulation, respectively, as a function of pulse width. The solid lines are fits described in the main text. (e) and (f) The SAW-amplitude dependence of the success rates. 
(of length $\tau_{\text {saw }}=0.36 \mathrm{~ns}$ ). As the number of cycles during a pulse of width $w_{p}$ is approximately $w_{p} / \tau_{\text {saw }}$, the success

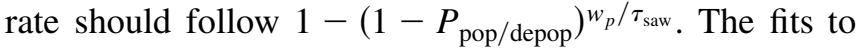
this equation are shown as the solid lines in Figs. 4(c) and 4(d), which give $P_{\text {pop }}=5 \times 10^{-5}$ and $P_{\text {depop }}=1 \times 10^{-5}$. These show that a SAW cycle needs to be repeated $10^{5}$ times to ensure one successful population/depopulation event. A drop in the success rate for population at longer pulse widths occurs as the electron that has already been added to the dot may be removed within the same pulse. Similarly, a drop in the success rate for depopulation can happen as the latter part of the same pulse may add an electron to the depopulated dot.

Figures 4(e) and 4(f) show the SAW-amplitude dependence of the success rate for the two transducers T1 and T2. The SAW amplitude (peak-to-peak amplitude of the electrostatic potential modulation) for each transducer is calibrated by measuring the conductance peak splitting (the details of this measurement have been published elsewhere [14]). The transducer power of $-2.1 \mathrm{dBm}$ on $\mathrm{T} 1$ used in the previous measurements corresponds to $7 \mathrm{mV}$ in SAW amplitude. Apart from a $\sim 1 \mathrm{mV}$ offset, which may be due to a calibration error, the two transducers show a similar behavior. These data show that at least $6 \mathrm{mV}$ SAW amplitude is necessary for population and depopulation. For depopulation, too large an amplitude leads to double depopulation $(+2 e)$.

The mechanism of population and depopulation is not yet fully understood. One might speculate that the SAW simply lowers and raises the entire dot potential below and above the Fermi energy, and many electrons enter and escape the dot during the pulse. However, the low probabilities per cycle deduced from the pulse-width dependence indicate that this is not the case. The process that we believe is happening is as follows. During a population pulse, the SAW lowers the dot and barrier potentials during every SAW cycle [see Fig. 2(a)]. When this occurs, electrons in the reservoir have an increased, but small, probability of tunneling into the dot. This tunneling may be to one of the excited states because the lowest unoccupied level is dropped well below the Fermi energy. After one electron has tunneled into the dot, the probability that another electron tunnels into the dot is suppressed if the electrochemical potential of the next available state (including the charging energy) is above the Fermi energy. If tunneling were not suppressed, double population could occur; however, this was not observed in these experiments. The depopulation process occurs when the dot potential is raised by the SAW. As the tunnel barrier is weaker at higher energy, the electron in the highest occupied state has a chance of escaping to the reservoir. Population and depopulation processes are likely to occur only through whichever of the two barriers is weaker if the dot potential is asymmetric, regardless of the directions of
SAW travel. This is consistent with the similarity in the SAW-amplitude dependence of the two transducers.

The minimum pulse width required for a population success rate $\sim 1$ was $20 \mu$ s with the setup shown. This may not be short enough for quantum-logic-gate applications. However, the required pulse width can be shortened either by using a larger SAW amplitude or by lowering the tunnel barrier. We note that pulses as short as 50 ns successfully depopulated the dot with different device settings.

We also note that it has been shown by other experiments that SAWs are capable of driving currents through a quantum dot $[17,18]$. The tunnel barriers of our dot are not transparent enough for this to happen. With continuous application of SAW pulses, we found that a transducer power of $5 \mathrm{dBm}$, much larger than used here, was needed to drive an acoustoelectric current through the dot.

In summary, we have demonstrated the use of surfaceacoustic-wave pulses for single-electron injection and extraction of an isolated quantum dot. In principle, a SAW pulse can be used to address many quantum dots on a chip if each dot can be independently tuned into Write or Read mode. Our approach can be used as an alternative to gate pulses in state preparation or read out in quantum-logicgate operations.

This research is part of QIP IRC (www.qipirc.org) (No. GR/S82176/01) and is supported by the UK EPSRC.

[1] M. A. Nielsen and I. L. Chuang, Quantum Computation and Quantum Information (Cambridge University Press, Cambridge, England, 2000).

[2] T. Fujisawa et al., Nature (London) 419, 278 (2002).

[3] T. Hayashi et al., Phys. Rev. Lett. 91, 226804 (2003).

[4] J. M. Elzerman et al., Nature (London) 430, 431 (2004).

[5] R. Hanson et al., Phys. Rev. Lett. 94, 196802 (2005).

[6] J. R. Petta et al., Science 309, 2180 (2005).

[7] D. Loss and D.P. DiVincenzo, Phys. Rev. A 57, 120 (1998).

[8] For example, cotunneling is considered to be a source of charge decoherence.

[9] M. Kataoka et al., J. Appl. Phys. 100, 063710 (2006).

[10] J. Cooper et al., Physica (Amsterdam) E6, 457 (2000).

[11] J. P. Pekola, A. B. Zorin, and M. A. Paalanen, Phys. Rev. B 50, 11255 (1994).

[12] M. Field et al., Phys. Rev. Lett. 70, 1311 (1993).

[13] U. Meirav, M. A. Kastner, and S. J. Wind, Phys. Rev. Lett. 65, 771 (1990).

[14] R. J. Schneble et al., Appl. Phys. Lett. 89, 122104 (2006).

[15] J. M. Shilton et al., J. Phys. Condens. Matter 8, L337 (1996); 8, L531 (1996).

[16] Although we do not know the exact value of the charging energy in this isolated regime, we estimate it to be around a few meV, as it is measured to be $1 \mathrm{meV}$ in the open regime where the dot should be larger.

[17] N.E. Fletcher et al., Phys. Rev. B 68, 245310 (2003).

[18] J. Ebbecke et al., Appl. Phys. Lett. 84, 4319 (2004). 